\title{
High-Speed Digital Coherent Receiver Based on Parallel Optical Sampling
}

Johannes Karl Fischer, Member, IEEE, Reinhold Ludwig, Lutz Molle, Carsten Schmidt-Langhorst, Member, OSA, Christoph Clemens Leonhardt, Andreas Matiss, and Colja Schubert

\begin{abstract}
We report a novel digital coherent receiver based on sampling the signal in the optical domain to overcome the speed limitations of electrical analog-to-digital converters. Digital coherent receivers with aggregate optical sampling rates of up to $112 \mathrm{GHz}$ are realized experimentally. The performance of such receivers is investigated in transmission experiments. Transmission of 56 GBd quadrature phase-shift keying signals over $610-\mathrm{km}$ standard single-mode fiber and coherent detection with electronic impairment mitigation is demonstrated. The employed electrical analog-to-digital converters have only $20 \mathrm{GHz}$ bandwidth and a sampling rate of $50 \mathrm{GHz}$.
\end{abstract}

Index Terms-Homodyne detection, optical fiber communication, optical receivers, signal sampling.

\section{INTRODUCTION}

C OHERENT receivers employing digital signal processing for electronic impairment mitigation and digital carrierphase recovery in conjunction with polarization-division multiplexing (PDM) and quadrature phase-shift keying (QPSK) have emerged as a favorable solution for $100 \mathrm{G}$ Ethernet transport [1]. However, scaling this solution to future Ethernet systems with a bit rate of $400 \mathrm{~Gb} / \mathrm{s}$ results in unfeasible requirements for the bandwidth and sampling rate of electrical analog-to-digital converters (ADC) [2]. Increasing the number of encoded bits per transmitted symbol relaxes these requirements, albeit at the cost of a significantly reduced tolerance to amplified spontaneous emission noise and nonlinear impairments.

Optical time-division demultiplexing of the received signal in a coherent receiver by using a pulsed local oscillator (LO) has been proposed as an approach to reduce the required ADC sampling rate [3]. Although impressive experimental results have been achieved with this technique [4]-[9], demonstrating bit rates exceeding $1 \mathrm{~Tb} / \mathrm{s}$ per wavelength carrier, it has major drawbacks. Coherent optical time-division demultiplexing requires

Manuscript received July 05, 2010; revised October 22, 2010; accepted October 22, 2010. Date of publication October 28, 2010; date of current version February 02, 2011. This work was supported in part by the German Federal Ministry of Education and Research in the program Optical Technologies under contract number 13N9356 and in part by the European Network of Excellence EURO-FOS.

J. K. Fischer, R. Ludwig, L. Molle, C. Schmidt-Langhorst, and C. Schubert are with the Fraunhofer Institute for Telecommunications, Heinrich-Hertz-Institute, 10587 Berlin, Germany (e-mail: johannes.fischer@hhi.fraunhofer.de).

C. C. Leonhardt and A. Matiss are with $\mathbf{u}^{2} \mathrm{t}$ Photonics AG, 10553 Berlin, Germany (e-mail: matiss@u2t.de).

Color versions of one or more of the figures in this paper are available online at http://ieeexplore.ieee.org

Digital Object Identifier 10.1109/JLT.2010.2090132 accurate synchronization of the received signal symbols with the LO pulses at the receiver, necessitating an optical clock recovery. The functionality of the optical clock recovery is susceptible to signal distortions due to chromatic dispersion (CD) and polarization-mode dispersion (PMD). If these distortions become too large, the optical clock recovery fails. Therefore, these impairments have to be compensated optically before clock recovery is performed. This, however, negates the main benefit of digital coherent receivers, which is adaptive electronic impairment mitigation.

Since a coherent detector using a pulsed LO is essentially an optical phase-sensitive sampler, coherent optical time-division demultiplexing can be interpreted as synchronous optical sampling with one sample per symbol. The concept of linear optical sampling by using a coherent receiver with a pulsed LO has been around for some time. In fact, optical sampling of highspeed optical signals by using a coherent homodyne receiver with short LO pulses has been experimentally demonstrated in [10], where eye diagrams of $80-\mathrm{Gb} / \mathrm{s}$ return-to-zero OOK signals were measured. Shortly after, the concept was generalized to measurements of constellation diagrams of phase-shift keying signals [11], [12]. However, the LO pulse repetition rate, i.e., the optical sampling rate was $10 \mathrm{MHz}$, which allows reconstruction of eye diagrams and constellation diagrams but is not sufficient for real-time detection of high-speed optical signals.

Parallel optical sampling was introduced in [13]-[15], where two parallel optical samplers with one-symbol delay between their sampling instants were used to measure differential phase diagrams of differential phase-shift keying signals. The LO repetition rate was also $10 \mathrm{MHz}$.

In principle, a combination of high LO pulse repetition rates as they are commonly used for coherent time-division demultiplexing and several parallel optical samplers allows real-time sampling of the received optical signal with an arbitrary number of samples per symbol. Such a receiver concept was outlined and verified in a configuration with two optical samplers yielding an aggregate optical sampling rate of $20 \mathrm{GHz}$ [16], [17].

Recently, we extended this concept and experimentally realized a receiver with four parallel optical samplers yielding up to $128 \mathrm{GHz}$ aggregate optical sampling rate [18], [19]. In [18], we used this receiver for optical sampling of signals having symbol rates up to $64 \mathrm{GBd}$ with two samples per symbol. In contrast to coherent optical time-division demultiplexing, no optical clock recovery is required and a free-running asynchronous $\mathrm{LO}$ can be used. Furthermore, CD and PMD compensation can be performed electronically such that the full functionality of serial digital coherent receivers is preserved. However, a major chal- 


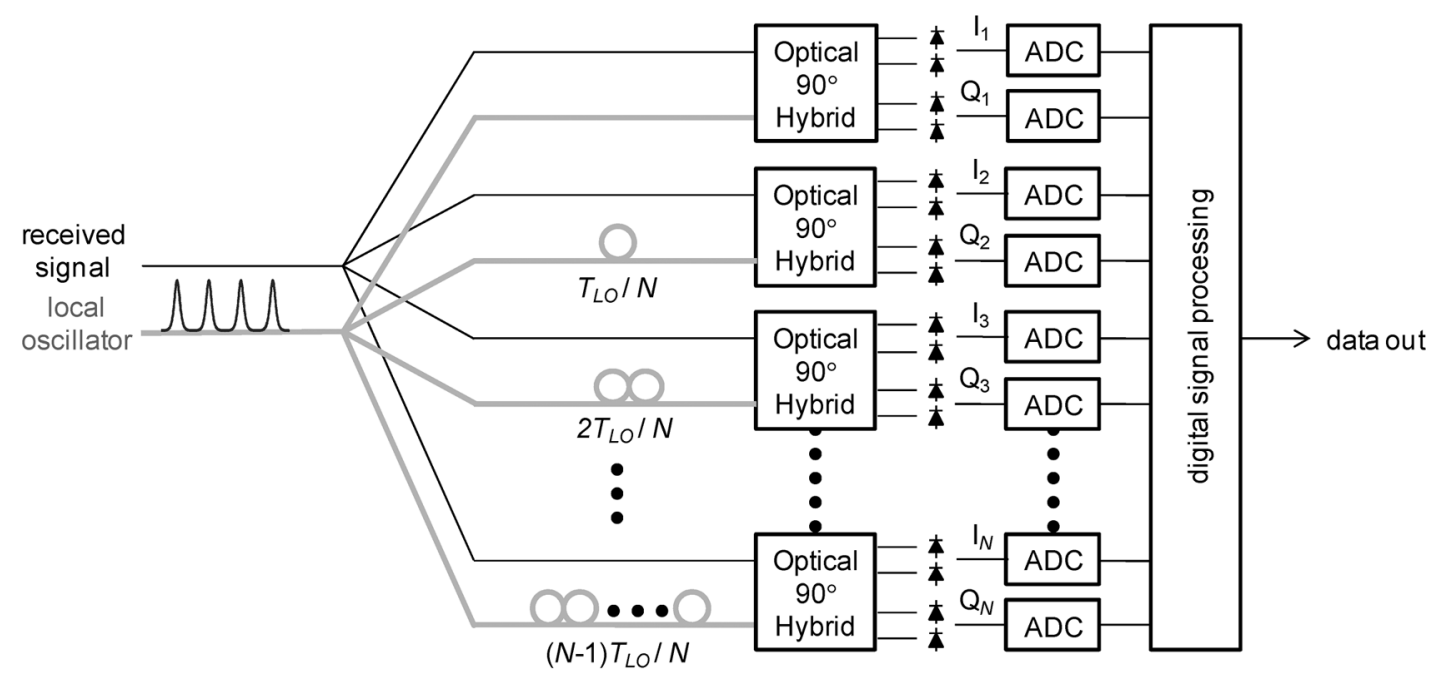

Fig. 1. Principle of coherent detection with parallel optical sampling.

lenge for a practical realization of such a parallel coherent receiver is the stabilization of the local oscillator phase at the parallel optical samplers, e.g., by photonic integration.

In this contribution, we discuss the principle of coherent detection based on parallel optical sampling and present results of transmission experiments with QPSK signals having symbol rates up to $64 \mathrm{GBd}$. In particular, transmission of $56 \mathrm{GBd}$ QPSK signals over 610-km standard single-mode fiber (SSMF) without optical dispersion compensation is demonstrated. Although the employed electrical ADCs have only $20 \mathrm{GHz}$ bandwidth and a sample rate of $50 \mathrm{GHz}$, coherent detection of the $56 \mathrm{GBd}$ QPSK signals is enabled by using four parallel delayed optical samplers yielding a total optical sampling rate of $112 \mathrm{GHz}$.

This paper is organized as follows. Section II reviews the basic principles enabling coherent detection by using parallel optical samplers and compares the required sampling rates of electrical ADCs for serial and parallel coherent receivers. Section III describes the setup of the transmission experiments as well as the employed integrated coherent receiver modules and the necessary modifications to the digital signal processing algorithms. Finally, the experimental results are discussed in Section IV.

\section{Coherent Detection With OpticAl SAMPling}

The principle of coherent detection in conjunction with parallel optical sampling was outlined in [17]. A block diagram of a receiver with a number of $N$ parallel optical samplers is shown in Fig. 1. The incoming optical signal and the LO are split into $N$ branches. In contrast to conventional coherent receivers, where continuous-wave light is used, a pulse train is required as an LO. To ensure equidistant sampling of the signal in the time domain, the $\mathrm{LO}$ in the $n$th branch is delayed by

$$
\tau_{n}=\frac{(n-1) T_{\mathrm{LO}}}{N}
$$

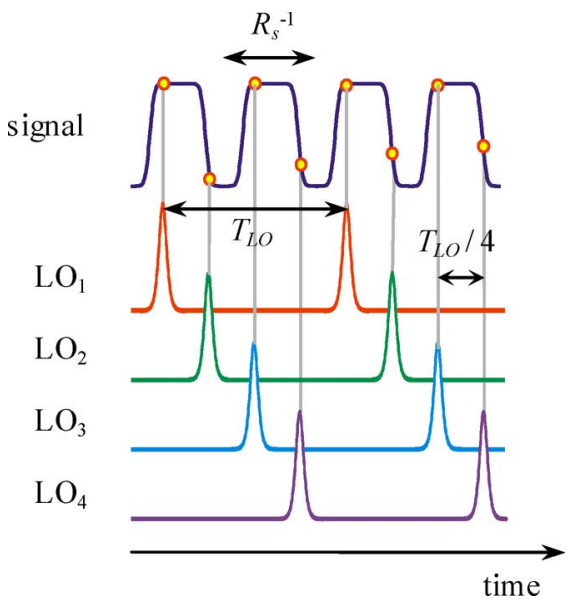

Fig. 2. Temporal alignment of LO pulses and signal in a configuration with four optical samplers ( $R_{s}$ : symbol rate).

where $T_{\mathrm{LO}}=R_{\mathrm{LO}}^{-1}$ is the time period between LO pulses and $R_{\mathrm{LO}}$ is the repetition rate of the LO pulses. Fig. 2 schematically shows the temporal alignment of LO pulse trains for a configuration with $N=4$ optical samplers. Signal and LO are then fed to $N$ parallel coherent front ends, each consisting of an optical $90^{\circ}$-hybrid and two balanced photodiodes. The photocurrents $I_{n}$ and $Q_{n}$ contain $I$ - and $Q$-components of the signal electric field sampled at time instants

$$
t_{n, k}=\tau_{n}+k T_{\mathrm{LO}} \quad \text { with } k=0,1,2, \ldots
$$

The analog photocurrents $I_{n}$ and $Q_{n}$ are then digitized by $2 N$ parallel electrical ADCs. Finally, electronic impairment compensation as well as digital carrier-phase recovery is performed in a digital signal processor. For polarization diversity, a receiver structure, as shown in Fig. 1, has to be used for each polarization tributary.

In the following two sections, we take a closer look on the required electrical bandwidth and sampling rate for serial and parallel coherent receiver schemes. 


\section{A. Serial Digital Coherent Receiver}

In a conventional digital coherent receiver, the electrical $\mathrm{ADCs}$ require a sampling rate

$$
f_{s, \mathrm{el}}=M_{\mathrm{el}} R_{s}
$$

where $R_{s}$ is the symbol rate of the received optical signal and $M_{\mathrm{el}}$ is the desired rational oversampling factor. According to the Nyquist-Shannon sampling theorem, an analog signal with a bandwidth of

$$
B_{\mathrm{el}}=\frac{f_{s, \mathrm{el}}}{2}
$$

can be sampled without aliasing, where a low-pass filter with rectangular transfer function of bandwidth $B_{\mathrm{el}}$ is assumed for simplicity. Both the required sampling rate and the electric bandwidth are thus proportional to the symbol rate of the received optical signal, and the proportionality factor is determined by the oversampling factor $M_{\mathrm{el}}$.

\section{B. Parallel Digital Coherent Receiver With Optical Sampling}

In a parallel digital coherent receiver according to Fig. 1, the received signal is optically sampled in each coherent front end. The required optical sampling rate per coherent front end is

$$
f_{s, \mathrm{opt}}=\frac{M_{\mathrm{opt}} R_{s}}{N}
$$

where $M_{\mathrm{opt}}$ is the desired rational oversampling factor.

Usually, the ADCs sample the photocurrents in an asynchronous fashion since distortions due to $\mathrm{CD}$ and PMD make a reliable clock recovery difficult. In that case, the required sampling rate of the ADCs becomes

$$
f_{s, \mathrm{el}}=M_{\mathrm{el}} f_{s, \mathrm{opt}}=\frac{M_{\mathrm{el}} M_{\mathrm{opt}} R_{s}}{N}
$$

and the electrical bandwidth follows from (4). However, the photocurrents $I_{n}$ and $Q_{n}$ have a strong frequency component at the LO repetition rate $R_{\mathrm{LO}}$ due to the optical sampling process. This enables recovery of the LO clock even for heavily impaired received signals. The recovered clock can be used to further reduce the required electrical sampling rate, since $M_{\mathrm{el}}=1$ becomes sufficient.

As an illustrative example, Fig. 3 shows the required sampling rate of the ADCs for up to 16 parallel optical samplers and optical symbol rates up to $200 \mathrm{GBd}$. While a configuration with four optical samplers effectively reduces the requirements for the ADCs, further parallelization yields only diminishing returns.

Considering possible single-carrier solutions for next generation $400 \mathrm{~Gb} / \mathrm{s}$ Ethernet systems such as $112 \mathrm{GBd}$ PDM-QPSK or 56 GBd PDM 16-ary quadrature amplitude modulation (QAM), a serial coherent receiver would require ADC sampling rates of 224 and $112 \mathrm{GHz}$, respectively (oversampling factor of two assumed) [2]. A parallel coherent receiver with four optical samplers could reduce that requirement to 56 and $28 \mathrm{GHz}$, respectively. ADCs with such sampling rates and a sufficient number of effective bits are commercially available today.

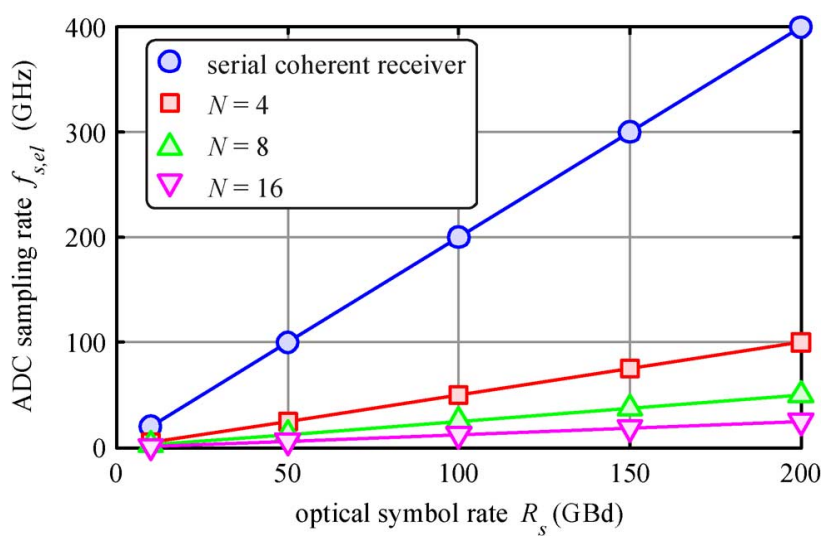

Fig. 3. Required ADC sampling rate for an oversampling factor of two and up to $N=16$ parallel optical samplers.

\section{EXPERIMENTAL SETUP}

In order to verify the concept of coherent detection with parallel optical sampling, we conducted two sets of experiments. First, we realized a coherent receiver based on two parallel optical samplers with an aggregate optical sampling rate of $64 \mathrm{GHz}$. With this receiver we were able to successfully detect 32-GBd single-polarization and 64-GBd alternate-polarization QPSK signals. In a second step, we extended the receiver to a configuration with four parallel optical samplers yielding a total optical sampling rate of $112 \mathrm{GHz}$. This allowed us to increase the detected symbol rate to $56 \mathrm{GBd}$ for single-polarization QPSK signals. For all configurations, we performed transmission experiments over $610 \mathrm{~km}$ SSMF without optical dispersion compensation. The dispersion was compensated using digital signal processing in the receiver. The following sections describe the experimental setup in detail.

\section{A. Transmitter}

The experimental setups of the two different transmitter configurations are shown in Fig. 4(a) and (b). An external cavity laser (ECL) with a linewidth of $100 \mathrm{kHz}$ emits continuous-wave light at a wavelength of $1550 \mathrm{~nm}$. The ECL is followed by an electroabsorption modulator (EAM) sinusoidally driven as a pulse carver. The pulses have a full-width at half-maximum of approximately 10 ps. The pulse train is QPSK modulated by an $I Q$-modulator (Fujitsu FTM7960EX) consisting of two dual-drive push-pull Mach-Zehnder modulators in a Mach-Zehnder superstructure. The inphase and quadrature components of the electric field are modulated independently by two pseudorandom binary sequences of length $2^{7}-1$, which are generated by an SHF 12100B bit-pattern generator (BPG). In order to decorrelate the sequences, the inverted data output of the BPG is delayed by about $1.4 \mathrm{~ns}$. The $I Q$-modulator is followed by an optical fiber delay multiplexer stage (MUX), where optical time-division multiplexing is performed. The multiplexer allows generation of single-polarization signals as well as symbol-interleaved alternate-polarization signals. Finally, the signal is filtered by an optical bandpass filter with a 3-dB bandwidth of $70 \mathrm{GHz}$.

The generation of the three considered symbol rates differs only in the settings of the optical fiber delay multiplexer stage 


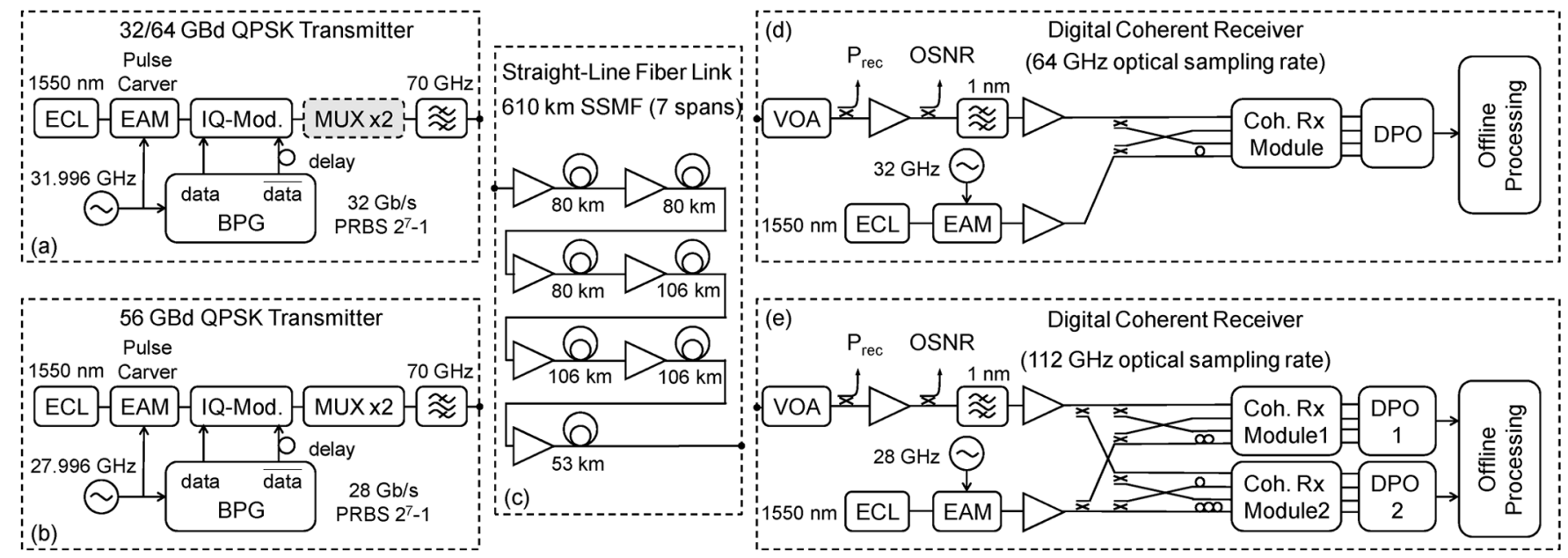

Fig. 4. Experimental setup. (a), (b) transmitter setups, (c) fiber link and (d), (e) receiver setups.

TABLE I

TRANSMITTER CONFIGURATIONS

\begin{tabular}{cccc}
\hline \hline $\begin{array}{c}\text { Symbol Rate } \\
(\mathrm{GBd})\end{array}$ & $\begin{array}{c}\text { Clock Frequency } \\
(\mathrm{GHz})\end{array}$ & $\begin{array}{c}\text { No. of MUX } \\
\text { stages }\end{array}$ & Polarization \\
\hline 32 & 31.996 & w/o MUX & $\begin{array}{c}\text { single- } \\
\text { polarization } \\
\text { single- } \\
\text { polarization } \\
\text { alternate- } \\
\text { polarization }\end{array}$ \\
56 & 27.996 & 1 & 1 (PolMux) \\
64 & 31.996 &
\end{tabular}

and the clock frequency of the electric driving signals for the EAM pulse carver and the BPG. The parameters are summarized in Table I.

\section{B. Fiber Link}

The setup of the 610-km fiber link is shown in Fig. 4(c). The fiber link comprises seven spans of SSMF $(3 \times 80 \mathrm{~km}+3 \times$ $106 \mathrm{~km}+53 \mathrm{~km}$ ). The loss of each span is compensated for by erbium-doped fiber amplifiers (EDFA). The launch power into each span is set to $2 \mathrm{dBm}$. No optical dispersion compensation is used. The residual chromatic dispersion after the fiber link is $10118 \mathrm{ps} / \mathrm{nm}$.

\section{Receiver}

The two employed receiver configurations are shown in Fig. 4(d) and (e). The optical signal-to-noise ratio (OSNR) is adjusted by using a variable optical attenuator and an EDFA preamplifier. The maximum available OSNR (measured with $0.1 \mathrm{~nm}$ resolution bandwidth) after the fiber link is approximately $20 \mathrm{~dB}$. After the preamplifier, the signal is filtered by a 1-nm optical bandpass filter and amplified by a second EDFA.

The LO pulses are generated by an ECL followed by an EAM pulse carver in a configuration similar to the transmitter pulse source. The ECL has a linewidth of $100 \mathrm{kHz}$. The optical emission frequencies of transmitter and LO ECL differ by approximately $410 \mathrm{MHz}$. The sinusoidally driven EAM pulse carver generates pulses with 10-ps full-width at half-maximum. The frequency of the sinusoid driving the EAM is adjusted to be
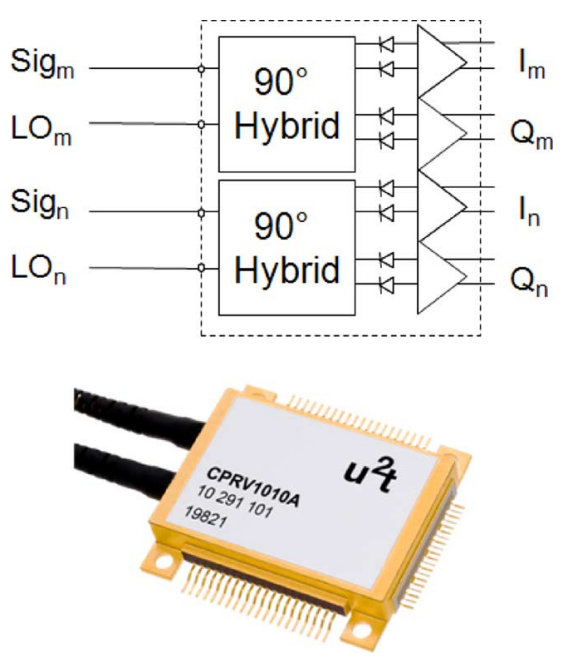

Fig. 5. Integrated coherent receiver module incorporating two coherent optical front ends and four differential transimpedance amplifiers.

$4 \mathrm{MHz}$ higher than the clock frequency used at the transmitter (cp. Table I). The differences in optical emission frequency and electrical clock frequency have been chosen deliberately but could be arbitrary within reasonable limits. The EAM is followed by an EDFA to adjust the LO power incident on the inputs of the coherent receiver modules.

In the following, we first discuss the receiver configuration with two parallel optical samplers and $64 \mathrm{GHz}$ optical sampling rate before discussing the extension to four optical samplers and $112 \mathrm{GHz}$ sampling rate.

The setup of the digital coherent receiver with $64 \mathrm{GHz}$ sampling rate is shown in Fig. 4(d). The repetition rate of the LO pulses is $R_{\mathrm{LO}}=32 \mathrm{GHz}$. Signal and $\mathrm{LO}$ are split by $3-\mathrm{dB}$ couplers into two branches. A delay of $T_{\mathrm{LO}} / 2$ is inserted in the lower $\mathrm{LO}$ branch to ensure equidistant sampling in the time domain. All branches are fed to an integrated coherent receiver module from $\mathrm{u}^{2} \mathrm{t}$ Photonics [20], [21]. A block diagram and a photograph of such a coherent receiver module are shown in Fig. 5. The module comprises two photonic integrated circuits 


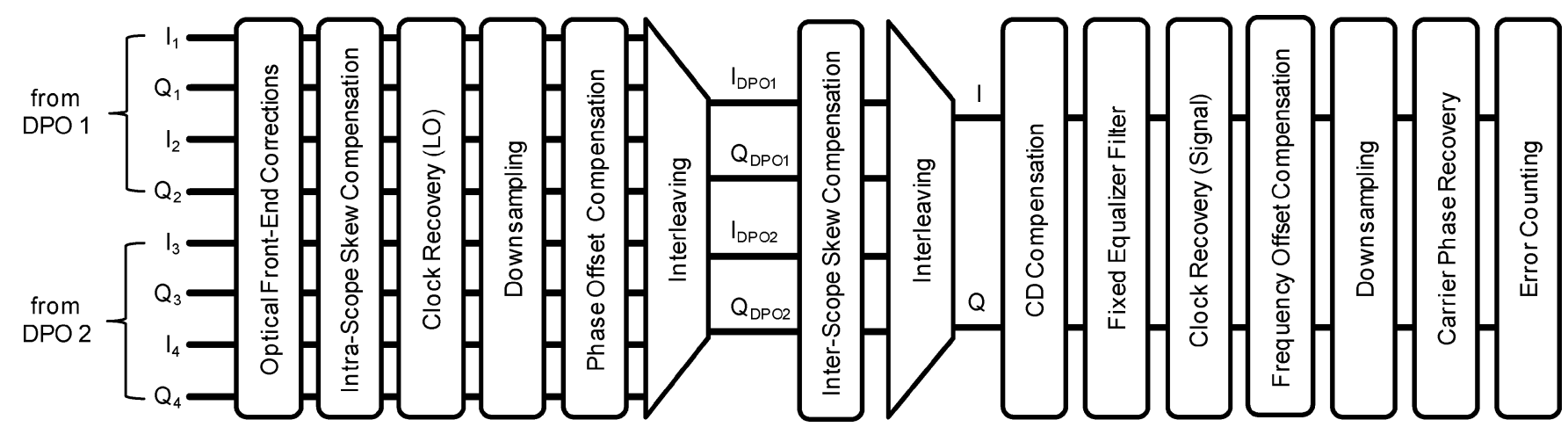

Fig. 6. Block diagram of the employed signal processing algorithms.

(PICs) copackaged with four differential transimpedance amplifiers. Per PIC, one optical $90^{\circ}$-hybrid is monolithically integrated with four waveguide $\mathrm{p}-\mathrm{i}-\mathrm{n}$ photodiodes in a balanced configuration.

The output voltages of the integrated coherent receiver module are asynchronously sampled and digitized by a Tektronix digital-phosphor oscilloscope (DPO) with four channels, a sampling rate of $50 \mathrm{GHz}$ per channel and $20 \mathrm{GHz}$ bandwidth. As explained in Section II-B, the required sampling rate of the electronic ADCs can be further reduced if the output voltages are sampled synchronously. Unfortunately, the employed DPO does not provide an option to externally clock its ADCs in order to set a desired sampling rate. Therefore, we use asynchronous electrical sampling. The received samples are then postprocessed offline in a computer to recover the QPSK data signal.

The setup of the digital coherent receiver with $112 \mathrm{GHz}$ sampling rate is shown in Fig. 4(e). The repetition rate of the LO pulses is $R_{\mathrm{LO}}=28 \mathrm{GHz}$. The receiver is further parallelized to achieve a higher sampling rate. Signal and LO are split into four branches, which are fed to two integrated coherent receiver modules. To ensure equidistant sampling of the optical signal, optical delays of $0.5 T_{\mathrm{LO}}, 0.25 T_{\mathrm{LO}}$, and $0.75 T_{\mathrm{LO}}$ are inserted in three of the four LO branches. This setup constitutes four delayed optical samplers, each with a sampling rate of $28 \mathrm{GHz}$, yielding a combined sampling rate of $112 \mathrm{GHz}$. In this case, two Tektronix DPOs are used for electronic analog-to-digital conversion of the output voltages of the two integrated coherent receiver modules.

For all measurements, the output power of the EDFAs is adjusted to yield a signal power of $-7 \mathrm{dBm}$ and an LO power of $3 \mathrm{dBm}$ at the input ports of the optical $90^{\circ}$-hybrids. The states of polarization of signal and LO are matched with manual polarization controllers.

\section{Offline Processing}

A block diagram of the employed signal-processing algorithms for the configuration with four optical samplers is shown in Fig. 6. The input signal consists of four sequences of complex samples $\mathrm{I}_{n}+j \mathrm{Q}_{n}(n \in\{1,2,3,4\})$ acquired by the two DPOs. Up to the first interleaving stage, all algorithms are performed for each of the four sample sequences individually. In principle, the signals can be further parallelized to be processed at lower rates [22].
In our experiments, imbalances of the optical front ends are corrected first. We use a Gram-Schmidt orthogonalization procedure to compensate for any quadrature imbalance of the optical $90^{\circ}$-hybrids [23]. After that, the timing skews between the channels of each DPO are compensated. Recovery of the LO clock allows a downsampling to a sampling rate $S_{R}=R_{\mathrm{LO}}$.

Since the network of feeding fibers to the optical $90^{\circ}$-hybrids is not phase stabilized, the relative phase between signal and LO drifts with time in the four branches. Taking one of the four sample sequences as a reference, there remain three phase offsets to be determined and compensated. We resort to a multidimensional optimization procedure reported in [24], which, however, is not suitable for implementation on a digital signal processor. A solution based on photonic integration of the feeding network together with the four optical $90^{\circ}$ hybrids and the photodiodes could completely avoid this problem. A smart phase tracking algorithm could also alleviate the problem. A first attempt to compensate a single phase offset with a tracking algorithm was proposed in [17]. However, the algorithm may fail depending on the transmitted bit pattern.

After compensation of the phase offset, the samples acquired by each DPO are interleaved in the time domain, yielding two sequences of complex samples: $I_{\mathrm{DPO} 1}+j \mathrm{Q}_{\mathrm{DPO} 1}$ (from DPO 1) and $I_{\mathrm{DPO} 2}+j \mathrm{Q}_{\mathrm{DPO} 2}$ (from $\mathrm{DPO} 2$ ).

Since the DPOs could not be triggered precisely enough to ensure that both DPOs start acquisition at the exact same time, extra measures had to be taken to compensate the timing skew between the samples acquired by DPO 1 and DPO 2. We used an additional EAM driven by an arbitrary waveform generator to periodically switch the LO ON and OFF during acquisition of the first 400 samples. The intensity (squared magnitude) of the acquired complex samples is shown in Fig. 7(a). Due to the periodic switching of the $\mathrm{LO}$, the interscope timing skew can be clearly seen as a time delay between the samples taken by DPO 1 and 2. In order to compensate for the interscope timing skew, the cross-correlation between the intensities of the samples acquired by DPO 1 and 2 is evaluated. The delay resulting in the maximum cross-correlation equals the interscope skew, which is then easily compensated. Fig. 7(b) shows the intensities after skew compensation, demonstrating precise matching of the correct sampling instants in the time domain.

Once the interscope timing skew is compensated, the samples acquired by DPO 1 and DPO 2 are interleaved and CD compen- 


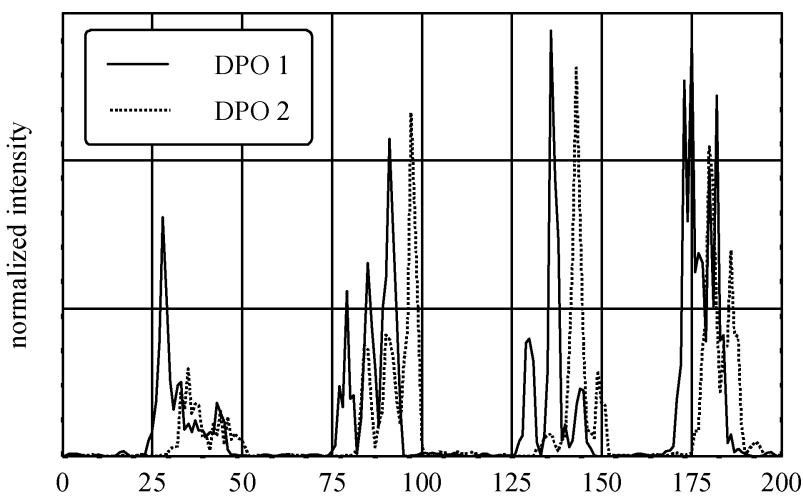

(a)

number of samples

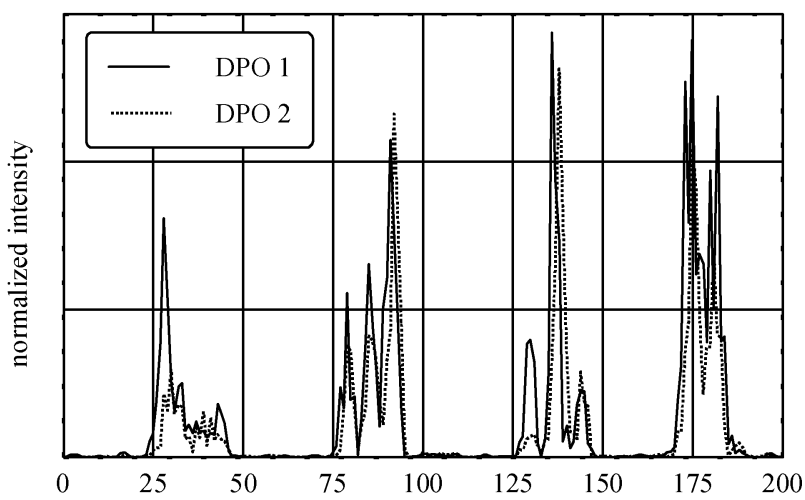

(b)

number of samples

Fig. 7. Intensity of the complex samples acquired by DPO 1 and DPO 2 (a) before and (b) after compensation of the interscope timing skew.

sation is performed in the frequency domain on the combined sequence of complex samples. A fixed equalizer filter is used to compensate for the frequency response of the optical front ends and the DPOs. After the signal clock has been recovered and the frequency offset between transmitter laser and LO laser is compensated, the signal is downsampled to one sample per symbol. The carrier phase is recovered by using a Viterbi-Viterbi algorithm [25], where the size of the moving-average window is optimized for each measurement. Error counting is performed on the recovered QPSK constellations to determine the bit-error ratio (BER).

\section{EXPERIMENTAL RESULTS}

The experimental results for a configuration with two optical samplers are shown in Fig. 8. Squares represent the measured back-to-back BER performance for 32 GBd QPSK signals. The required OSNR for a $\mathrm{BER}=10^{-3}$ (abbreviated as ROSNR in the following) is $\sim 15.2 \mathrm{~dB}$. After transmission over the $610-\mathrm{km}$ fiber link, a small penalty of about $0.5 \mathrm{~dB}$ is observed (triangles). This result demonstrates that electronic CD compensation can be performed when an adequate optical sampling rate is used (in our experiments we used two times oversampling). Furthermore, the polarization tributaries of a 64-GBd alternate-polarization QPSK signal (circles) are successfully received. The measured ROSNR is $\sim 19.4 \mathrm{~dB}$. That is about $3.7 \mathrm{~dB}$ more than the ROSNR of the $32 \mathrm{GBd}$ signals, i.e., there is a $0.7 \mathrm{~dB}$ excess penalty resulting from polarization multiplexing.

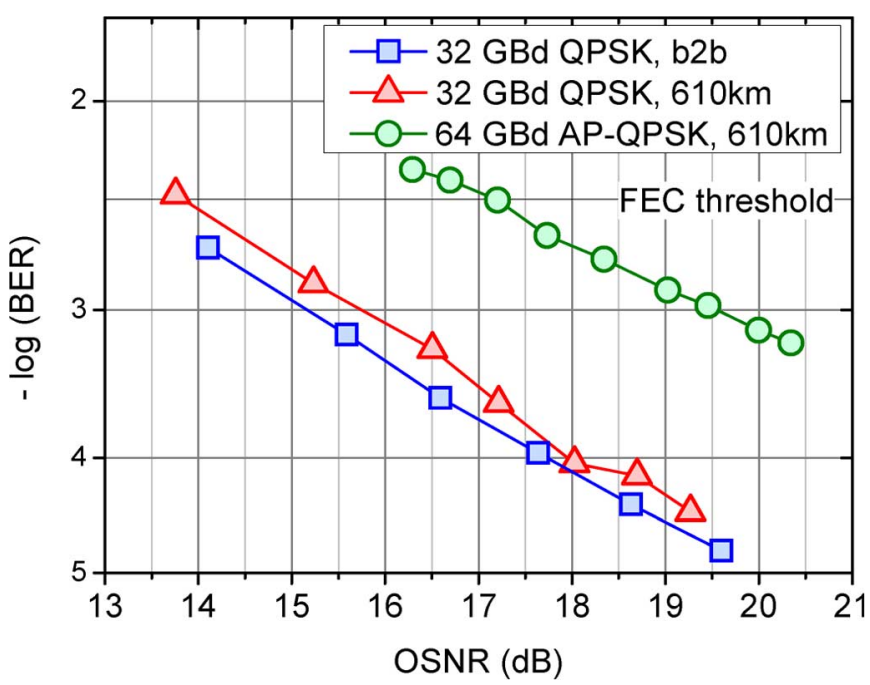

Fig. 8. Experimental results for a configuration with two optical samplers.

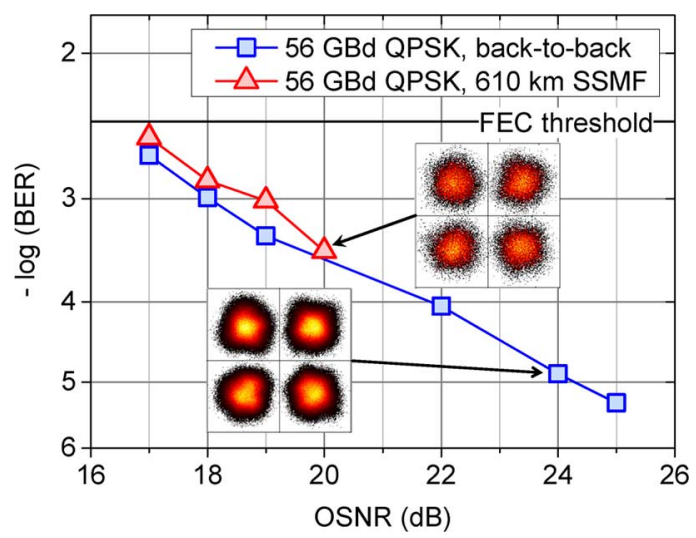

Fig. 9. Experimental results for a configuration with four optical samplers. Insets show constellation diagrams back-to-back and after transmission.

In a second experiment, single-polarization 56 GBd QPSK signals are generated, transmitted, and received. The results are shown in Fig. 9. The left inset of Fig. 9 shows the received constellation diagram (representing $\sim 10^{6}$ symbols) for BER $=$ $1.3 \times 10^{-5}$ at $24 \mathrm{~dB}$ OSNR. The back-to-back ROSNR for the $56 \mathrm{GBd}$ QPSK signals is $18 \mathrm{~dB}$. This is comparable to experimental results reported in [26], which were achieved with a serial coherent receiver using ADCs with $30 \mathrm{GHz}$ bandwidth and $80 \mathrm{GHz}$ sampling rate. Since this is still far off the theoretical limit of $13.3 \mathrm{~dB}$ [27], there is still plenty of potential for improvement.

Transmission of the $56 \mathrm{GBd}$ signals over $610 \mathrm{~km}$ results in a small penalty of less than $1 \mathrm{~dB}$. The additional penalty compared to transmission of 32 GBd QPSK signals is likely due to nonideal compensation of the three phase offsets, since a residual phase offset leads to incomplete compensation of the CD. At the maximum available OSNR after the fiber link (about $20 \mathrm{~dB}$ ), a BER $=3.4 \times 10^{-4}$ is achieved. The right inset of Fig. 9 shows the received constellation diagram at this configuration (representing $\sim 10^{5}$ symbols). BERs well below the threshold of $3.8 \times 10^{-3}$ for forward error correction (FEC) with $7 \%$ overhead were achieved with all considered configurations. 


\section{CONCLUSION}

Coherent detection using parallel optical sampling overcomes the limitation of the optical symbol rate imposed by the bandwidth of receiver electronics and the sampling rate of electrical ADCs. The proposed architecture is scalable by further parallelization to accommodate arbitrarily high-optical symbol rates as long as the pulsewidth of the LO pulses is sufficiently small. The achievable degree of parallelization is mainly limited by the amount of optical components that can be integrated on a single opto-electronic integrated circuit.

In contrast to systems using coherent optical time-division demultiplexing, this coherent receiver scheme needs no optical clock recovery and preserves the full functionality of serial digital coherent receivers, i.e., adaptive electronic compensation of chromatic dispersion and PMD in the receiver.

Using a receiver configuration with $64 \mathrm{GHz}$ optical sampling rate, we demonstrate transmission of $32 \mathrm{GBd}(64 \mathrm{~Gb} / \mathrm{s})$ single-polarization and $64 \mathrm{GBd}(128 \mathrm{~Gb} / \mathrm{s})$ alternate-polarization QPSK signals over $610-\mathrm{km}$ standard single-mode fiber without optical dispersion compensation.

Parallel optical sampling with a sampling rate of $112 \mathrm{GHz}$ enables coherent detection of $56 \mathrm{GBd}(112 \mathrm{~Gb} / \mathrm{s})$ single-polarization QPSK signals as well as subsequent electronic impairment mitigation despite employing electrical ADCs having only $20 \mathrm{GHz}$ bandwidth and a sampling rate of $50 \mathrm{GHz}$. The $56 \mathrm{GBd}$ QPSK signals are also transmitted over 610-km standard singlemode fiber without optical dispersion compensation, achieving a BER performance well below the FEC threshold.

The enabling techniques in our experiments are optical signal sampling with two samples per symbol using an unsynchronized pulsed LO and integrated coherent receiver modules based on optical $90^{\circ}$-hybrids monolithically integrated with balanced detectors in a compact package.

\section{REFERENCES}

[1] C. R. S. Fludger, T. Duthel, D. van den Borne, C. Schulien, E.-D. Schmidt, T. Wuth, J. Geyer, E. De Man, G.-D. Khoe, and H. de Waardt, "Coherent equalization and POLMUX-RZ-DQPSK for robust 100-GE transmission," J. Lightw. Technol., vol. 26, no. 1, pp. 64-72, Jan. 2008.

[2] T. Pfau, "Hardware requirements for coherent systems beyond 100G," presented at the 35th Eur. Conf. Opt. Commun., Vienna, Austria, Sep. 2009 [Online]. Available: http://conference.vde.com/ecoc-2009/programs/Pages/SundayWorkshops.aspx

[3] C. Zhang, Y. Mori, K. Igarashi, K. Katoh, and K. Kikuchi, "Ultrafast operation of digital coherent receivers using their time-division demultiplexing function ," J. Lightw. Technol., vol. 27, no. 3, pp. 224-232, Feb. 2009.

[4] K. Kikuchi, K. Igarashi, Y. Mori, and C. Zhang, "Demodulation of 320-Gbit/s optical quadrature phase-shift keying signal with digital coherent receiver having time-division demultiplexing function," presented at the Opt. Fiber Commun. Conf. , San Diego, CA, Mar. 2008, Paper OTuO4.

[5] C. Zhang, Y. Mori, K. Igarashi, and K. Kikuchi, "Demodulation of 640Gbit/s polarization-multiplexed OTDM QPSK signals using a digital coherent receiver," presented at the Opt. Fiber Commun. Conf., San Diego, CA, Mar. 2008, Paper PDP6.

[6] C. Zhang, Y. Mori, K. Igarashi, K. Katoh, and K. Kikuchi, "Demodulation of 480-Gbit/s 8PSK OTDM signal with digital coherent receiver," presented at the 34th Eur. Conf. Opt. Commun., Brussels, Belgium, Sep. 2008, Paper Mo.4.D.6.
[7] C. Zhang, Y. Mori, K. Igarashi, K. Katoh, and K. Kikuchi, "Demodulation of 1.28-Tbit/s polarization-multiplexed 16-QAM signals on single carrier with digital coherent receiver," presented at the Opt. Fiber Commun. Conf., San Diego, CA, Mar. 2009, Paper OTuG3.

[8] C. Schmidt-Langhorst, R. Ludwig, D.-D. Groß, L. Molle, M. Seimetz, R. Freund, and C. Schubert, "Generation and coherent time-division demultiplexing of up to $5.1 \mathrm{~Tb} / \mathrm{s}$ single-channel 8-PSK and 16-QAM signals," presented at the Opt. Fiber Commun. Conf., San Diego, CA, Mar. 2009, Paper PDPC6.

[9] C. Schmidt-Langhorst, R. Ludwig, L. Molle, D.-D. Groß, R. Freund, and C. Schubert, "Terabit/s single-carrier transmission systems based on coherent time-division demultiplexing," presented at the Opt. Fiber Commun. Conf., San Diego, CA, Mar. 2010, Paper OThV3.

[10] C. Dorrer, D. C. Kilper, H. R. Stuart, G. Raybon, and M. G. Raymer, "Linear optical sampling," IEEE Photon. Technol. Lett., vol. 15, no. 12, pp. 1746-1748, Dec. 2003.

[11] C. Dorrer, C. R. Doerr, I. Kang, R. Ryf, J. Leuthold, and P. J. Winzer, "Measurement of eye diagrams and constellation diagrams of optical sources using linear optics and waveguide technology," J. Lightw. Technol., vol. 23, no. 1 , pp. 178-186, Jan. 2005.

[12] C. Dorrer, "Monitoring of optical signals from constellation diagrams measured with linear optical sampling," J. Lightw. Technol., vol. 24, no. 1, pp. 313-321, Jan. 2006.

[13] K. Okamoto and F. Ito, "Ultrafast measurement of optical DPSK signals using 1-symbol delayed dual-channel linear optical sampling," IEEE Photon. Technol. Lett., vol. 20, no. 11 , pp. 948-950, Jun. 2008 .

[14] K. Okamoto and F. Ito, "Dual-channel linear optical sampling for simultaneously monitoring ultrafast intensity and phase modulation," $J$. Lightw. Technol., vol. 27, no. 12, pp. 2169-2175, Jun. 2009.

[15] K. Okamoto and F. Ito, "Nearly shot-noise-limited performance of dual-channel linear optical sampling for ultrafast DPSK signals," IEEE J. Quantum Electron., vol. 45, no. 6, pp. 711-719, Jun. 2009.

[16] X. Chen, I. Kim, G. Li, H. Zhang, and B. Zhou, "Coherent detection using optical time-domain sampling," presented at the Opt. Fiber Commun. Conf., San Diego, CA, Mar. 2008, Paper JThA62.

[17] X. Chen, X. Xie, I. Kim, G. Li, H. Zhang, and B. Zhou, "Coherent detection using optical time-domain sampling,", IEEE Photonics Technol. Lett., vol. 21, no. 5, pp. 286-288, Mar. 2009.

[18] J. K. Fischer, R. Ludwig, L. Molle, C. Schmidt-Langhorst, A. Galperin, T. Richter, C. C. Leonhardt, A. Matiss, and C. Schubert, "High-speed digital coherent receiver with parallel optical sampling," presented at the Opt. Fiber Commun. Conf., San Diego, CA, Mar. 2010, Paper PDPB4.

[19] J. K. Fischer, R. Ludwig, L. Molle, C. Schmidt-Langhorst, C. C. Leonhardt, A. Matiss, and C. Schubert, "Digital coherent receiver based on parallel optical sampling," presented at the 36th Eur. Conf. Optical Communication, Torino, Italy, Sep. 2010, Paper Th.10.A.4.

[20] A. Matiss, R. Ludwig, J. K. Fischer, L. Molle, C. Schubert, C. C. Leonhardt, H.-G. Bach, R. Kunkel, and A. Umbach, "Novel integrated coherent receiver module for $100 \mathrm{G}$ serial transmission," presented at the Optical Fiber Communication Conf., San Diego, CA, Mar. 2010, Paper PDPB3.

[21] R. Ludwig, J. K. Fischer, A. Matiss, L. Molle, C. C. Leonhardt, H.-G. Bach, R. Kunkel, A. Umbach, and C. Schubert, "Performance of a novel integrated coherent receiver module for $100 \mathrm{G}$ Ethernet applications and beyond," presented at the 36th Eur. Conf. Optical Communication, Torino, Italy, Sep. 2010.

[22] R. Noé, "PLL-free synchronous QPSK polarization multiplex/diversity receiver concept with digital I\&Q baseband Processing," Photon. Technol. Lett., vol. 17, no. 4, pp. 887 -889, Apr. 2005.

[23] I. Fatadin, S. J. Savory, and D. Ives, "Compensation of quadrature imbalance in an optical QPSK coherent receiver," IEEE Photon. Technol. Lett., vol. 20, no. 20, pp. 1733-1735, Oct. 2008.

[24] L. D. Coelho, O. Gaete, and N. Hanik, "An algorithm for global optimization of optical communication systems," Int. J. Electron. Commun., vol. 63, no. 7, pp. 541-550, Jul. 2009.

[25] D.-S. Ly-Gagnon, S. Tsukamoto, K. Katoh, and K. Kikuchi, "Coherent detection of optical quadrature phase-shift keying signals with carrier phase estimation ," J. Lightw. Technol., vol. 24, no. 1, pp. 12-21, Jan. 2006.

[26] P. J. Winzer, A. H. Gnauck, G. Raybon, M. Schnecker, and P. J. Pupalaikkis, "56-Gbaud PDM-QPSK: Coherent detection and 2 500-km transmission," presented at the 35th Eur. Conf. Optical Communication, Vienna, Austria, Sep. 2009, Paper PD2.7.

[27] F. Xiong, Digital Modulation Techniques. Norwood, MA: Artech House, 2006, ch. 4. 
Johannes Karl Fischer (S'04-M'09) was born in Berlin, Germany, in 1976. He studied electrical engineering at the Technische Universität Berlin in Berlin, Germany, and the University of Manchester, Manchester, U.K. In 2003 and 2009, he received the Dipl.-Ing. and the Dr.-Ing. degrees (both with distinction) in electrical engineering from the Technische Universität, Berlin, Germany.

$\mathrm{He}$ is currently a Research Associate at the Fraunhofer Institute for Telecommunications, Heinrich-Hertz-Institute, Berlin, Germany. His research interests include advanced modulation formats, coherent detection, and fiber nonlinearity.

Dr. Fischer is a member of the Verband der Elektrotechnik Elektronik Informationstechnik. He received a best student paper award of the Asia-Pacific Optical Communications Conference in 2005.

Reinhold Ludwig was born in Lahnstein, Germany, in 1952. He received the Ing.-Grad. degree from the Fachhochschule Koblenz, Remagen, Germany, in 1974, and the Dipl.-Ing. and Dr.-Ing. degrees from the Technical University Berlin, Berlin, Germany, in 1985 and 1993, respectively.

In 1985, he joined the Heinrich-Hertz-Institut (HHI), Berlin, Germany, where he is currently involved in research on photonic components and systems. In 1991, he was a Visiting Scientist at Nippon Telephone and Telegraph Company and was at Bell Labs in 1993. Since 1985, he has authored or coauthored more than 300 scientific papers and has been the holder of several patents. In 1996, he founded the first HHI spin-off company (LKF Advanced Optics GmbH) and served as the Chief Executive Officer until the merger of LKF and u2t Innovative Optoelectronic Components $\mathrm{GmbH}$ in 2001.

Dr. Ludwig is a member of the Verband der Elektrotechnik Elektronik Informationstechnik. In 1999, his group received the Philip Morris Research Award, and he was nominated for the Innovation Award of the German Bundespraesident.

Lutz Molle received the Dipl.-Ing. degree from the Department of Electrical Engineering, Technical University of Berlin, Berlin, Germany, in 2004.

Since 2005, he has been with the Fraunhofer Institute for Telecommunications, Heinrich-Hertz-Institut, Department Photonic Networks and Systems, Berlin, Germany. His current research interests include the field of long-haul system design and high-speed upgrades of submarine and metro transmission systems using advanced modulation formats.

Carsten Schmidt-Langhorst was born in Berlin, Germany, in 1972. He received the Dipl.-Phys. and the Ph.D. degrees (Dr.Rer.Nat.) in physics from the Technical University of Berlin, Berlin, Germany, in 1997 and 2004, respectively.

Since 1998, he has been with the Fraunhofer Institute for Telecommunications, Heinrich-Hertz-Institute, Berlin, Germany, where he has been engaged in research on transmission, all-optical processing, and detection of optical data signals at a picosecond timescale, and, in particular, all-optical sampling techniques. He is currently heading several projects in the field of ultrafast optical transmission technology.

Dr. Schmidt-Langhorst is a member of the Optical Society of America, the Deutsche Physikalische Gesellschaft, and the Verband der Elektrotechnik Elektronik Informationstechnik. He received the Philip Morris Research Award in 1999.
Christoph Clemens Leonhardt was born in Berlin, on January 16, 1975. He studied electrical engineering at the Beuth University of Applied Science, Berlin, Germany, where he received the Diploma degree (Dipl. Ing. FH) in 2001.

In 2001, he joined $\mathbf{u}^{2} \mathrm{t}$ Photonics AG, Berlin, Germany, as a Development Engineer in the R\&D Department. The focus of his thesis was the time and frequency behavior of 50-GHz photodetectors under optical high power conditions. He is responsible for several $40 \mathrm{~Gb} / \mathrm{s}$ photoreceiver development projects and ultrahigh-speed optoelectronic components.

Andreas Matiss was born in Krefeld, Germany, on March 14, 1978. He studied electrical engineering at the University of Duisburg-Essen, Germany, where he received the Diploma degree (Dipl.-Ing.) in 2003. He received the Ph.D. (Dr.Ing.) degree from the University of Duisburg-Essen, Essen, Germany, in 2008.

In 2003, he joined the Solid-State-Electronics Department, Center for Semiconductor Technology and Optoelectronics, where he was engaged in research on ultrafast resonant tunneling diode structures for electrical and optoelectronic applications in high-speed telecommunication systems. Since 2008, he has been with $\mathbf{u}^{2} \mathrm{t}$ Photonics AG, Berlin, Germany, where he is currently a Project Manager in the R\&D Department being responsible for the development of ultrahigh-speed integrated optoelectronic components.

Dr. Matiss was awarded the ITG-Sponsorship-Award by the German Information Technology Society (VDE|ITG) in 2009.

Colja Schubert was born in Berlin, Germany, in 1973. He received the Dipl.Phys. and Dr.rer.nat. degrees in physics from the Technische Universität, Berlin, Germany, in 1998 and 2004, respectively.

From 1996 to 1997, he was an exchange student at the Strathclyde University, Glasgow, U.K. During his diploma thesis in 1997-1998, he was with the Max-Born Institute for Nonlinear Optics and Short Pulse Spectroscopy, Berlin, Germany. Since 2000, he has been a member of the scientific staff with the Fraunhofer Institute for Telecommunications, Heinrich-Hertz-Institute, Berlin, Germany, where he is engaged in research on high-speed transmission systems and all-optical signal processing. He is also currently the Head of the Submarine \& Core Systems Group of the Department of Photonic Networks and Systems.

Dr. Schubert is a member of the German Physical Society. 